Original scientific paper - Izvorni znanstveni rad

UDK: 637.112

\title{
The differences in heat stress resistance due to dairy cattle breed
}

doi: $10.15567 /$ mljekarstvo.2017.0203

\author{
Vesna Gantner ${ }^{1}$, Tina Bobićl ${ }^{*}$, Maja Gregićl ${ }^{1}$ Ranko Gantner ${ }^{1}$, \\ Krešimir Kuterovac ${ }^{2}$, Klemen Potočnik ${ }^{3}$ \\ ${ }^{1}$ J.J. Strossmayer University of Osijek, Faculty of Agriculture, \\ Kralja Petra Svačića 1d, 31000 Osijek, Croatia \\ ${ }^{2}$ Inagra Ltd, J.J. Strossmayera 341, 31000 Osijek, Croatia \\ ${ }^{3}$ University of Ljubljana, Biotechnical faculty, Department of Animal Science, \\ Groblje 3, Domžale, Slovenia
}

Received - Prispjelo: 22.07.2016. Accepted - Prihvaćeno: 06.04.2017.

\begin{abstract}
Considering the rapid climate changes worldwide, with the purpose to reduce financial losses for dairy farmers and enable a more sustainable farming as well, there is an increasing necessity to implement breeding values for heat resistance in breeding strategies. The estimation of breeding values requires a determination of temperature-humidity index (THI) threshold value. Therefore, the objective of this study was to determine the temperature-humidity index (THI) threshold values for daily milk yield, daily fat and protein content of milk produced by Holsteins and dairy Simmentals in Croatian farms. With that purpose, 1.070,554 test-day records from 70.135 Holsteins reared on 5.679 farms and 1.300,683 test-day records from 86.013 Simmentals reared on 8.827 farms in Croatia were compared to records of ambient temperature and relative humidity collected in the barn, during regular milking recordings from January 2005 to December 2012. Accordingly the following conclusions could be drawn: threshold values for daily milk yield were highly dependant on the parity and breed (Holsteins: 68, 69 and 72 for $1^{\text {st }}, 2^{\text {nd }}, 3+$ parity; Simmentals: 77 for $1^{\text {st }}$ ); daily fat and protein contents showed a highly significant decrease due to heat stress condition (THI in 66-80) in both, Holstein and Simmental cows, and in all parity classes; multiparous cows were more resistant to heat stress than primiparous; Simmental cows were more resistant to heat stress than Holsteins.
\end{abstract}

Key words: dairy cattle, Holstein, Simmental, heat stress, threshold value

\section{Introduction}

The consequences of climate change become, in the last few decades, more and more expressed worldwide. In accordance with the Intergovernmental Panel on Climate Change (IPCC, 2007), in the near future dairy cattle will be exposed to unfavourable climatic conditions in regions that now are not characterised by climate extremes. In accordance with this forecast, Reiczigel et al. (2009) determined in Hungary an increase in the number of heat stress days/year (temperature-humidity index
THI $>68$ ) from 5 to 17 in the last 30 years. Also, Gauly et al. (2013) found that when considering the scenarios of global warming, heat stress of highyielding dairy cows became an increasing concern of dairy producers in Europe. Dunn et al. (2014) concluded that the number of days exceeding the THI threshold in southern parts of the UK could increase from on average 1-2 per year to over 20 per year by 2100 . Worldwide, dairy cattle are kept in various production and housing systems (Nardone et al., 2010).

${ }^{*}$ Corresponding author/Dopisni autor: E-mail: tbobic@pfos.hr 
Indoor housing systems applied for dairy cattle breeding require, to the preservation of optimal microclimate conditions in the barn for achieving the productive potential of individual cows. The interrelation between ambient temperature and relative humidity is important for guaranteeing a good level of animal welfare, reproduction and profitability of the dairy farm. Any extreme combination is potentially harmful. In environment with low temperature and high humidity, cows increase heat production and consume more feed in order to compensate body energy losses. When the animal is overheated, high humidity may lead to infections of the respiratory tract or the udder. On the other hand, high temperature and low relative humidity may dehydrate mucous membranes thus increasing vulnerability to viruses and bacteria (Romaniuk and Overby, 2005). The combination of high temperature and high relative humidity was proven to have the most detrimental effect through inducing heat stress in cows. Under heat stress conditions, lactating cows tend to reduce their dry matter intake (DMI) and milk production (West et al., 1999). Moreover, heat stress is associated with changes in milk composition, increase in somatic cell counts (SCC) and mastitis frequencies (Bouraoui et al., 2002; Collier et al., 2012; Correa-Calderon et al., 2004; Ravagnolo et al., 2000; St-Pierre et al., 2003; West, 2003). Additionally, a deteriorating effect on reproductive performances was also observed (Bohmanova et al., 2007; Ravagnolo et al., 2000). Numerous studies showed that high producing cows were much more susceptible to heat stress than low producing cows (Bohmanova, 2006; Collier et al. 2006). Considering the intensive genetic selection for milk production, Kadzere et al. (2002) assumed that the cow physiology of thermoregulation changed. High producing cows had larger frames and larger gastrointestinal tracts which allow digestion of more feed, resulting in an increase in metabolic heat, which in turn reduces the cow's ability to maintain normal temperature at unfavourable conditions. Finally, an increase in milk yield, feed intake, and metabolic heat shifts the thermoneutrality to lower temperatures. Berman (2005) stated that a shift in the daily milk yield from 35 to $45 \mathrm{~kg} / \mathrm{d}$ leads to a higher sensitivity to thermal stress and reduces the threshold temperature for intermediate heat stress by $5^{\circ} \mathrm{C}$.

The most common measure of heat stress in dairy cows is the temperature-humidity index (THI) which is a combination of ambient temperature and relative humidity and is a useful and easy way to assess the risk of heat stress (Kibler, 1964). Du Preez et al. (1990 a, b) determined that milk production and feed intake were affected by heat stress if THI values were higher than 72. Bouraoui et al. (2002) put the threshold at 69, while Bernabucci et al. (2010) as well as Collier et al. (2012) suggested the threshold being at 68. Vitali et al. (2009) assumed that the risk of cow's death started to increase when THI reached 80. A significant decrease in the daily milk traits (yield and contents) was also determined in Croatian environmental conditions with highest decline during summer period in Eastern and Mediterranean Croatia (Gantner et al., 2011). In numerous dairy-producing areas worldwide heat stress conditions represent a significant financial burden. For example in the USA it was estimated to be between $\$ 897$ million and $\$ 1.500$ million per year (St-Pierre et al., 2003). In recent years the milk production in the European Union milk production has moved to Atlantic regions characterised by a less intensive farming, more land suitable for pasture and a milder climate (GIRA, 2012). Forecasts indicate that this movements will continue unless heat stress effect in the south eastern Europe were minimised by applying appropriate methods. There are many methods to decrease the impact of heat stress including the shading, cooling, nutrition (Kadzere et al., 2002; West, 2003) and selection for heat stress resistance (Bohmanova, 2006). Ravagnolo et al. (2000) found an antagonistic relationship between cow's production and heat tolerance implying a deteriorate effect of the selection for productivity on cow's resistance to heat stress. The unfavourable genetic relationship between THI and productive and reproductive traits was found in few studies (Ravagnolo and Misztal, 2002a,b; Freitas et al., 2006; Aguilar et al., 2009). On the other hand, the presence of high yielding Holstein cows in Israel demonstrated a good example that selective production could be successful in terms of heat stress (Aharoni et al., 1999). Implementation of breeding values for heat resistance in breeding strategies would certainly reduce financial losses of dairy farmers and enable sustainable farming. Estimation of breeding values requires determination of THI threshold values. Therefore, the objective of this paper was to determine THI threshold values for daily milk traits (yield and content) of dairy cows (Holsteins and Simmentals) on Croatian farms. 


\section{Materials and methods}

Individual test-day records of Holstein and Simmental dairy cows were collected during routine milking recordings using an alternate milking recording method from January 2005 to December 2012 in Croatia and were used for the analysis. Monthly, at each recording, milk yields were measured during the evening or the morning milkings in an alternate way. Logical control of milk data was performed according to ICAR standards (2003). Additionally, at each recording, ambient temperature and relative humidity in the barns were recorded. Daily temperature-humidity index (THI) was calculated using the equation by Kibler (1964):

$$
\mathrm{THI}=1.8 \times \mathrm{Ta}-(1-\mathrm{RH}) \times(\mathrm{Ta}-14.3)+32
$$

where $\mathrm{Ta}$ is average temperature in ${ }^{\circ} \mathrm{C}$ and $\mathrm{RH}$ relative humidity as a fraction of the unit.
Records with lactation stage in $<6$ days and $>500$ days, age at first calving in $<21$ and $>36$ months, missing or parity $>6$ for Holstein and $>7$ for Simmental cows, and missing or nonsense Ta and RH value were deleted from the dataset. Regarding the parity, cows were divided into 3 groups: $1^{\text {st }}, 2^{\text {nd }}$ and $3+(\geq 3)$. Also, only cows with $\min 3$ test day per parity were taken into analysis. Data were provided by the Croatian Agricultural Agency, after passing a logical control consisting of 1.070.554 test-day records from 70.135 Holsteins reared on 5.679 farms and 1.300683 test-day records from 86.013 Simmentals reared on 8.827 farms in Croatia. Variability of milk traits (daily milk yield, DMY; daily fat content, DFC; daily protein content, DPC) per parity $\left(1^{\text {st }}, 2^{\text {nd }}, 3+\right)$ and breed (Holstein, Simmental) is presented in Table 1.

The THI threshold values for daily milk traits were determined by a least square analyses of variance for each given THI value (from 66 to 80 ) in regard to the parity class $\left(1^{\text {st }}, 2^{\text {nd }}, 3+\right.$ ) and breed (Holstein, Simmental) using the PROC MIXED procedure in SAS (SAS Institute Inc., 2000). Following mixed model was used:

$y_{i j k l m n}=\mu+b_{1}\left(d_{i} / 305\right)+b_{2}\left(d_{i} / 305\right)^{2}+b_{3} \ln \left(305 / d_{i}\right)+b_{4} \ln ^{2}\left(305 / d_{i}\right)+S_{j}+A_{k}+R_{l}+T_{m}+e_{i j k l m n}$

Where $\mathrm{y}_{\mathrm{ijklm}}=$ estimated daily milk trait (milk yield, fat/protein content);

$\mu=$ intercept;

$\mathrm{b}_{1}, \mathrm{~b}_{2}, \mathrm{~b}_{3}, \mathrm{~b}_{4}=$ regression coefficients;

$\mathrm{d}_{\mathrm{i}}=$ days in milk ( $\mathrm{i}=6$ to 500 day);

$S_{j}=$ fixed effect of calving season class $j(j=1 / 2005$ to $12 / 2012)$;

$A_{k}=$ fixed effect of age at calving class $\mathrm{k}\left(\mathrm{k}=21\right.$ to 36 month)*only for $1^{\text {st }}$ parity,

$\mathrm{R}_{\mathrm{l}}=$ fixed effect of region $\mathrm{k}(\mathrm{l}=$ Croatian counties $)$,

$\mathrm{T}_{\mathrm{m}}=$ fixed effect of THI class ( $\mathrm{m}=0$ (normal condition - values under the given threshold) or 1 (heat stress condition - values equal and above the given threshold)),

$\mathrm{e}_{\mathrm{ijklm}}=$ residual.

The significance of differences between the THI classes were tested by Scheffe's method of multiple comparisons. The threshold value was defined as the lowest THI value at which significant differences in milk traits was determined.

Table 1. Descriptive statistics of milk traits (daily milk yield, DMY; daily fat content, DFC; daily protein content, DPC) per parity and breed

\begin{tabular}{ccccccccccc}
\hline \multirow{2}{*}{ Trait } & \multirow{2}{*}{ Breed/Parity } & \multicolumn{3}{c}{$1^{\text {st }}$} & \multicolumn{3}{c}{$2^{\text {nd }}$} & \multicolumn{3}{c}{$3+$} \\
\cline { 3 - 10 } & & mean & SD & $n$ & mean & SD & $n$ & mean & SD & $\mathrm{n}$ \\
\hline \multirow{2}{*}{ DMY } & Holstein & 19.97 & 7.19 & 389021 & 21.82 & 9.27 & 296613 & 21.62 & 9.42 & 355212 \\
\cline { 2 - 11 } & Simmental & 15.06 & 5.16 & 324899 & 15.98 & 6.12 & 289317 & 15.90 & 6.15 & 641689 \\
\hline \multirow{2}{*}{ DFC } & Holstein & 4.15 & 0.92 & 392136 & 4.20 & 0.97 & 293411 & 4.17 & 0.97 & 351232 \\
\cline { 2 - 10 } & Simmental & 4.21 & 0.85 & 326606 & 4.22 & 0.90 & 289495 & 4.14 & 0.91 & 639508 \\
\hline \multirow{2}{*}{ DPC } & Holstein & 3.41 & 0.47 & 394401 & 3.44 & 0.48 & 295321 & 3.38 & 0.48 & 353648 \\
\cline { 2 - 10 } & Simmental & 3.45 & 0.46 & 328775 & 3.50 & 0.47 & 291639 & 3.45 & 0.46 & 645464 \\
\hline
\end{tabular}




\section{Results and discussion}

Analysis of the microclimate parameters recorded in the barns in the summer season (June, July, August) during the period 2005-2012 are presented in Figure 1 (Holsteins) and Figure 2 (Simmentals). During the months of June, July and August, mean ambient temperature varied from 21 to $23.5^{\circ} \mathrm{C}$, from 23 to $28^{\circ} \mathrm{C}$ and from 22 to $28^{\circ} \mathrm{C}$, respectively. Taking into account that the upper limit of cow's comfort zone was $25^{\circ} \mathrm{C}$ (Yousef, 1985), even mean values of measured ambient temperatures in Holstein's barns indicated a prevalence of heat stress conditions. When maximum daily ambient temperatures were considered, the lowest measured value was $35^{\circ} \mathrm{C}$. Combination of these high temperatures with high relative humidity values were able to cause heat stress conditions during the entire summer period.

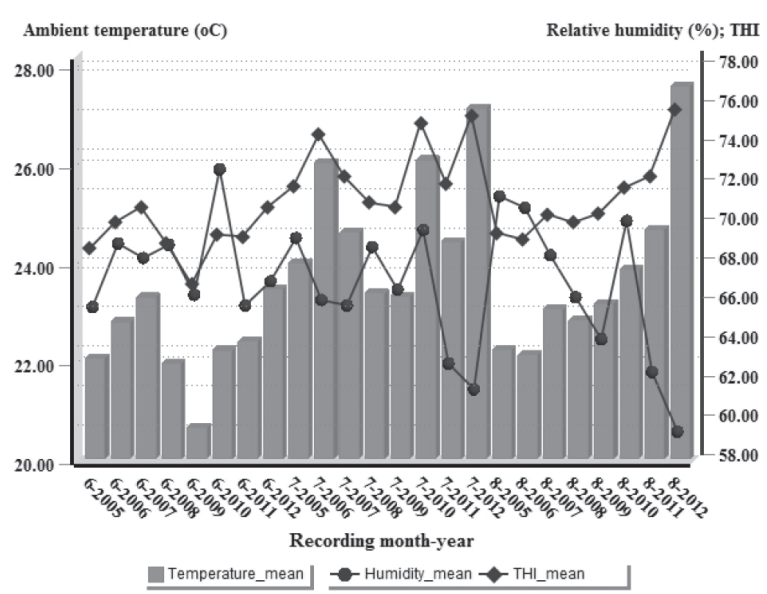

Similar mean values of indoor environmental parameters were also determined in the barns with Simmental cows and, compared to the previous ones, a higher prevalence of days with extreme conditions (ambient temperature $=40{ }^{\circ} \mathrm{C}$, relative humidity $>96 \%$, as well as THI $>96$ ) was found.

Least square means regarding the fixed effect of THI class $(0,1)$ on daily milk yield in accordance to the parity class $\left(1^{\text {st }}, 2^{\text {nd }}, 3+\right)$ and breed (Holstein, Simmental) are shown in Table 2. Environmental conditions in the barns with THI values equal to 68 caused significant, but small $(0.075 \mathrm{~kg} /$ day $)$ drop in daily production of first parity Holsteins. Highly significant decrease of daily milk yield was observed when THI value in the barns was above 68 , when the estimated drop in milk yield was between 0.159 and $0.329 \mathrm{~kg} /$ day. The highest decrease was determined in environmental condition characterised by THI equal to 77.

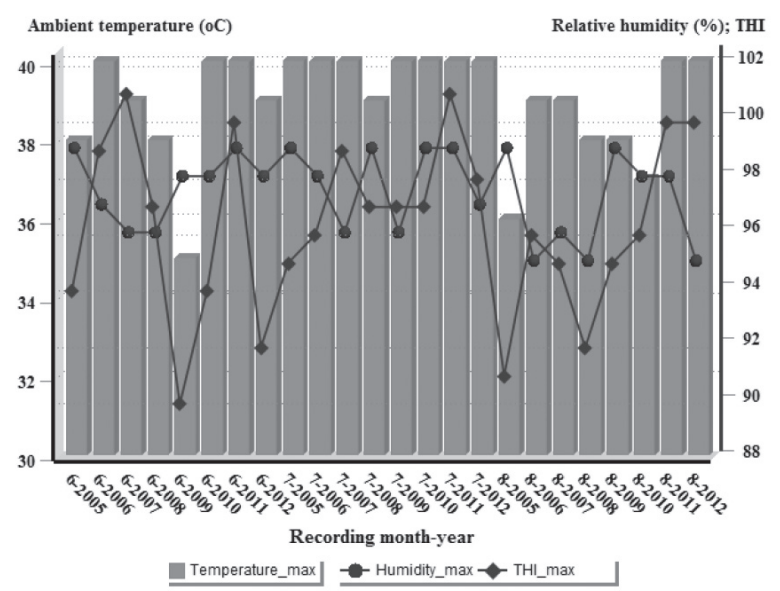

Figure 1. Mean and maximum daily ambient temperature, relative humidity and THI values measured during milk recording of Holstein cows
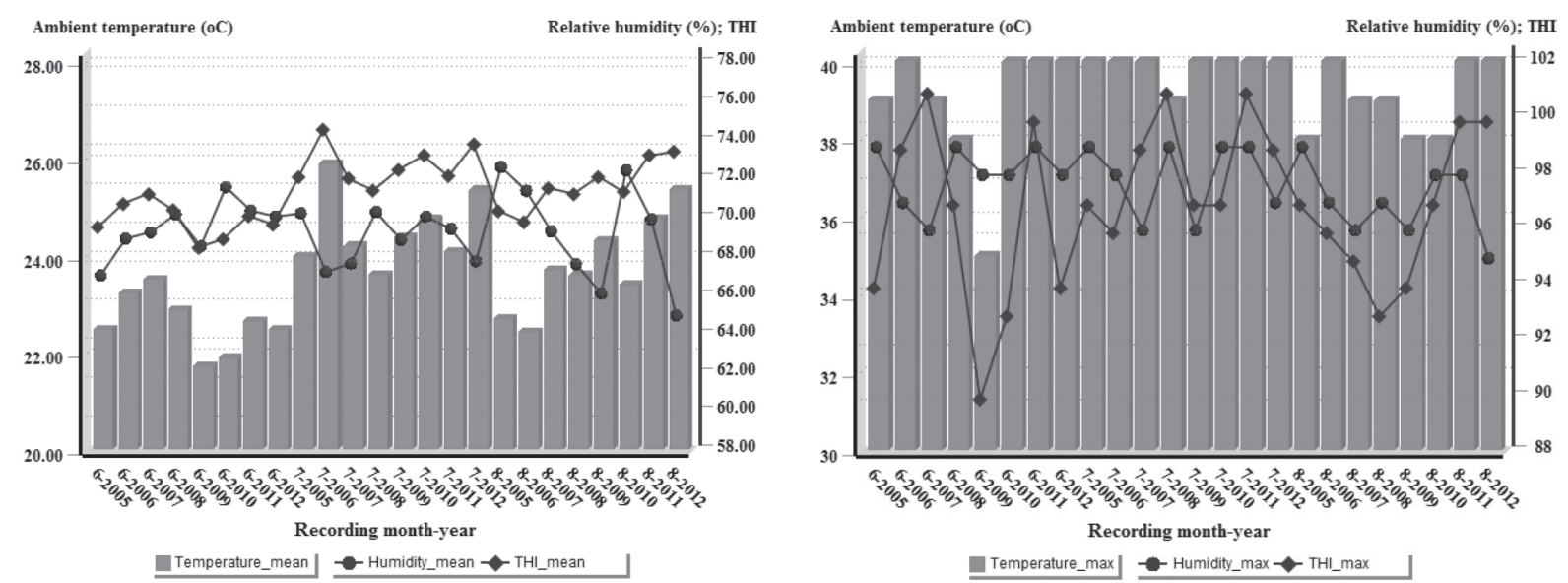

Figure 2. Mean and maximum daily ambient temperature, relative humidity and THI values measured during milk recording of Simmental cows 
Table 2. Least square means of daily milk yield regarding the given threshold values in accordance to the parity class and breed

\begin{tabular}{|c|c|c|c|c|c|c|}
\hline \multirow[b]{2}{*}{ ThHo } & \multicolumn{3}{|c|}{ Holstein } & \multicolumn{3}{|c|}{ Simmental } \\
\hline & Ls0 & Lsl & Difference & Ls0 & Ls1 & Difference \\
\hline & \multicolumn{6}{|c|}{$1^{\text {st }}$ parity } \\
\hline THI66 & $19.62 \pm 0.048$ & $19.64 \pm 0.050$ & $-0.016 \pm 0.023^{\mathrm{ns}}$ & $14.60 \pm 0.048$ & $14.64 \pm 0.050$ & $-0.037 \pm 0.018^{*}$ \\
\hline THI67 & $19.62 \pm 0.048$ & $19.63 \pm 0.051$ & $-0.005 \pm 0.024^{\mathrm{ns}}$ & $14.60 \pm 0.048$ & $14.65 \pm 0.050$ & $-0.052 \pm 0.019^{* *}$ \\
\hline THI68 & $19.64 \pm 0.048$ & $19.57 \pm 0.051$ & $0.075 \pm 0.024^{* * *}$ & $14.61 \pm 0.048$ & $14.64 \pm 0.050$ & $-0.037 \pm 0.019^{*}$ \\
\hline THI69 & $19.66 \pm 0.048$ & $19.50 \pm 0.051$ & $0.159 \pm 0.025^{* * *}$ & $14.61 \pm 0.048$ & $14.63 \pm 0.051$ & $-0.025 \pm 0.020^{\mathrm{ns}}$ \\
\hline THI70 & $19.66 \pm 0.048$ & $19.48 \pm 0.052$ & $0.180 \pm 0.026^{* * *}$ & $14.61 \pm 0.048$ & $14.65 \pm 0.051$ & $-0.046 \pm 0.021^{* *}$ \\
\hline THI71 & $19.66 \pm 0.048$ & $19.44 \pm 0.053$ & $0.219 \pm 0.027^{* * *}$ & $14.61 \pm 0.048$ & $14.66 \pm 0.052$ & $-0.050 \pm 0.022^{* *}$ \\
\hline THI72 & $19.66 \pm 0.048$ & $19.40 \pm 0.054$ & $0.268 \pm 0.029^{* * *}$ & $14.61 \pm 0.048$ & $14.64 \pm 0.052$ & $-0.025 \pm 0.023^{\mathrm{ns}}$ \\
\hline THI73 & $19.66 \pm 0.048$ & $19.40 \pm 0.054$ & $0.261 \pm 0.031^{* * *}$ & $14.61 \pm 0.048$ & $14.66 \pm 0.053$ & $-0.055 \pm 0.025^{* *}$ \\
\hline THI74 & $19.66 \pm 0.048$ & $19.36 \pm 0.056$ & $0.298 \pm 0.033^{* * *}$ & $14.61 \pm 0.048$ & $14.65 \pm 0.054$ & $-0.041 \pm 0.026^{\mathrm{ns}}$ \\
\hline THI75 & $19.64 \pm 0.048$ & $19.46 \pm 0.057$ & $0.182 \pm 0.035^{* * *}$ & $14.61 \pm 0.048$ & $14.62 \pm 0.055$ & $-0.011 \pm 0.028^{\mathrm{ns}}$ \\
\hline THI76 & $19.64 \pm 0.048$ & $19.43 \pm 0.059$ & $0.218 \pm 0.038^{* * *}$ & $14.61 \pm 0.048$ & $14.62 \pm 0.056$ & $-0.002 \pm 0.031^{\mathrm{ns}}$ \\
\hline THI77 & $19.65 \pm 0.048$ & $19.32 \pm 0.061$ & $0.329 \pm 0.041^{* * *}$ & $14.62 \pm 0.048$ & $14.55 \pm 0.058$ & $0.069 \pm 0.034^{*}$ \\
\hline THI78 & $19.64 \pm 0.048$ & $19.40 \pm 0.065$ & $0.238 \pm 0.047^{* * *}$ & $14.62 \pm 0.048$ & $14.55 \pm 0.060$ & $0.069 \pm 0.038$ \\
\hline THI79 & $19.62 \pm 0.047$ & $19.61 \pm 0.069$ & $0.014 \pm 0.053^{\mathrm{ns}}$ & $14.61 \pm 0.048$ & $14.58 \pm 0.063$ & $0.031 \pm 0.042^{\mathrm{ns}}$ \\
\hline \multirow[t]{2}{*}{ THI80 } & $19.62 \pm 0.047$ & $19.86 \pm 0.074$ & $-0.241 \pm 0.059^{* * *}$ & $14.62 \pm 0.048$ & $14.55 \pm 0.066$ & $0.069 \pm 0.047^{\mathrm{ns}}$ \\
\hline & \multicolumn{6}{|c|}{$2^{\text {nd }}$ parity } \\
\hline THI66 & $21.73 \pm 0.081$ & $21.88 \pm 0.084$ & $-0.154 \pm 0.031^{* * *}$ & $15.51 \pm 0.059$ & $15.73 \pm 0.061$ & $-0.216 \pm 0.021^{* * *}$ \\
\hline THI67 & $21.74 \pm 0.081$ & $21.83 \pm 0.084$ & $-0.090 \pm 0.032^{* * *}$ & $15.51 \pm 0.059$ & $15.73 \pm 0.061$ & $-0.220 \pm 0.022^{* * *}$ \\
\hline THI68 & $21.76 \pm 0.081$ & $21.77 \pm 0.084$ & $-0.001 \pm 0.033^{\mathrm{ns}}$ & $15.52 \pm 0.059$ & $15.74 \pm 0.061$ & $-0.219 \pm 0.023^{* * *}$ \\
\hline THI69 & $21.78 \pm 0.081$ & $21.68 \pm 0.085$ & $0.103 \pm 0.034^{* * *}$ & $15.53 \pm 0.059$ & $15.75 \pm 0.062$ & $-0.223 \pm 0.024^{* * *}$ \\
\hline THI70 & $21.79 \pm 0.081$ & $21.62 \pm 0.086$ & $0.176 \pm 0.036^{* * *}$ & $15.53 \pm 0.059$ & $15.75 \pm 0.062$ & $-0.223 \pm 0.025^{* * *}$ \\
\hline THI71 & $21.79 \pm 0.081$ & $21.65 \pm 0.086$ & $0.140 \pm 0.037^{* * *}$ & $15.54 \pm 0.059$ & $15.76 \pm 0.063$ & $-0.225 \pm 0.026^{* * *}$ \\
\hline THI72 & $21.79 \pm 0.081$ & $21.60 \pm 0.087$ & $0.189 \pm 0.040^{* * *}$ & $15.54 \pm 0.059$ & $15.73 \pm 0.064$ & $-0.188 \pm 0.028^{* * *}$ \\
\hline THI73 & $21.79 \pm 0.081$ & $21.57 \pm 0.088$ & $0.220 \pm 0.042^{* * *}$ & $15.55 \pm 0.059$ & $15.73 \pm 0.064$ & $-0.182 \pm 0.029^{* * *}$ \\
\hline THI74 & $21.79 \pm 0.081$ & $21.55 \pm 0.090$ & $0.239 \pm 0.045^{* * *}$ & $15.55 \pm 0.059$ & $15.73 \pm 0.065$ & $-0.178 \pm 0.031^{* * *}$ \\
\hline THI75 & $21.77 \pm 0.081$ & $21.67 \pm 0.091$ & $0.104 \pm 0.048^{* *}$ & $15.55 \pm 0.059$ & $15.76 \pm 0.066$ & $-0.206 \pm 0.034^{* * *}$ \\
\hline THI76 & $21.78 \pm 0.080$ & $21.63 \pm 0.093$ & $0.143 \pm 0.052^{* *}$ & $15.55 \pm 0.059$ & $15.76 \pm 0.068$ & $-0.205 \pm 0.037^{* * *}$ \\
\hline THI77 & $21.79 \pm 0.080$ & $21.45 \pm 0.096$ & $0.339 \pm 0.057^{* * *}$ & $15.56 \pm 0.059$ & $15.65 \pm 0.070$ & $-0.093 \pm 0.041^{*}$ \\
\hline THI78 & $21.78 \pm 0.080$ & $21.55 \pm 0.100$ & $0.228 \pm 0.063^{* * *}$ & $15.56 \pm 0.059$ & $15.66 \pm 0.073$ & $-0.096 \pm 0.045^{\circ}$ \\
\hline THI79 & $21.77 \pm 0.080$ & $21.64 \pm 0.105$ & $0.125 \pm 0.071^{*}$ & $15.56 \pm 0.059$ & $15.66 \pm 0.076$ & $-0.102 \pm 0.050^{*}$ \\
\hline \multirow[t]{2}{*}{ THI80 } & $21.76 \pm 0.080$ & $21.81 \pm 0.111$ & $-0.052 \pm 0.079^{\mathrm{ns}}$ & $15.56 \pm 0.059$ & $15.70 \pm 0.080$ & $-0.136 \pm 0.055^{\circ}$ \\
\hline & \multicolumn{6}{|c|}{$3+$ parity } \\
\hline THI66 & $21.67 \pm 0.047$ & $21.83 \pm 0.052$ & $-0.152 \pm 0.029^{* * *}$ & $15.55 \pm 0.065$ & $15.78 \pm 0.066$ & $-0.226 \pm 0.014^{* * *}$ \\
\hline THI67 & $21.68 \pm 0.047$ & $21.81 \pm 0.052$ & $-0.131 \pm 0.030^{* * *}$ & $15.56 \pm 0.065$ & $15.79 \pm 0.066$ & $-0.231 \pm 0.015^{* * *}$ \\
\hline THI68 & $21.70 \pm 0.047$ & $21.77 \pm 0.053$ & $-0.067 \pm 0.031^{*}$ & $15.56 \pm 0.065$ & $15.79 \pm 0.066$ & $-0.226 \pm 0.015^{* * *}$ \\
\hline THI69 & $21.72 \pm 0.047$ & $21.68 \pm 0.054$ & $0.045 \pm 0.032^{\mathrm{ns}}$ & $15.57 \pm 0.065$ & $15.79 \pm 0.066$ & $-0.218 \pm 0.016^{* * *}$ \\
\hline THI70 & $21.72 \pm 0.047$ & $21.68 \pm 0.054$ & $0.040 \pm 0.033^{\mathrm{ns}}$ & $15.57 \pm 0.065$ & $15.80 \pm 0.066$ & $-0.225 \pm 0.017^{* * *}$ \\
\hline THI71 & $21.72 \pm 0.047$ & $21.67 \pm 0.055$ & $0.050 \pm 0.035^{\mathrm{ns}}$ & $15.58 \pm 0.065$ & $15.81 \pm 0.067$ & $-0.227 \pm 0.017^{* * *}$ \\
\hline THI72 & $21.73 \pm 0.047$ & $21.59 \pm 0.057$ & $0.143 \pm 0.037^{* * *}$ & $15.59 \pm 0.065$ & $15.79 \pm 0.067$ & $-0.205 \pm 0.018^{* * *}$ \\
\hline THI73 & $21.73 \pm 0.047$ & $21.61 \pm 0.058$ & $0.115 \pm 0.039^{* *}$ & $15.59 \pm 0.065$ & $15.81 \pm 0.067$ & $-0.223 \pm 0.020^{* * *}$ \\
\hline THI74 & $21.73 \pm 0.047$ & $21.55 \pm 0.060$ & $0.178 \pm 0.042^{* * *}$ & $15.59 \pm 0.065$ & $15.79 \pm 0.068$ & $-0.199 \pm 0.021^{* * *}$ \\
\hline THI75 & $21.72 \pm 0.047$ & $21.69 \pm 0.062$ & $0.030 \pm 0.045^{\mathrm{ns}}$ & $15.60 \pm 0.065$ & $15.77 \pm 0.068$ & $-0.168 \pm 0.022^{* * *}$ \\
\hline THI76 & $21.71 \pm 0.047$ & $21.73 \pm 0.065$ & $-0.015 \pm 0.049^{\mathrm{ns}}$ & $15.60 \pm 0.065$ & $15.73 \pm 0.069$ & $-0.123 \pm 0.024^{* * *}$ \\
\hline THI77 & $21.72 \pm 0.047$ & $21.61 \pm 0.069$ & $0.113 \pm 0.054^{*}$ & $15.61 \pm 0.065$ & $15.68 \pm 0.070$ & $-0.069 \pm 0.027^{* *}$ \\
\hline THI78 & $21.71 \pm 0.047$ & $21.68 \pm 0.073$ & $0.033 \pm 0.059^{\mathrm{ns}}$ & $15.61 \pm 0.065$ & $15.69 \pm 0.071$ & $-0.081 \pm 0.030^{* *}$ \\
\hline THI79 & $21.71 \pm 0.047$ & $21.81 \pm 0.079$ & $-0.103 \pm 0.067^{\mathrm{ns}}$ & $15.61 \pm 0.065$ & $15.72 \pm 0.072$ & $-0.119 \pm 0.033^{* * *}$ \\
\hline THI80 & $21.71 \pm 0.047$ & $21.86 \pm 0.087$ & $-0.151 \pm 0.075^{*}$ & $15.61 \pm 0.065$ & $15.72 \pm 0.074$ & $-0.115 \pm 0.037^{* *}$ \\
\hline
\end{tabular}

* ThHo - given threshold value; 0 - class under, and 1 - class above the given threshold value 
The Holsteins in second lactation were slightly more heat resistant and experienced significant decrease of daily milk yield when $\mathrm{THI}=69$. Similarly, like first parity cows, Holsteins in second parity also had the highest drop in daily milk yield (equal to $0.339 \mathrm{~kg} /$ day) when $\mathrm{THI}=77$. The Holsteins in third and higher lactation, as expected, were much more resistant to the heat stress conditions. These cows experienced significant drop of daily milk production when $\mathrm{THI}=72$. Although, the drop in daily milk yield was determined even in older cows, the amount of the decrease was quite smaller comparing to the cows in first and second parity indicating that resistance to heat stress increases with cow's age.

Within the analyses of the Simmental first parity cows, the initial significant drop in daily milk yield was determined for THI in the barns at 77 . In older Simmentals (second and higher lactations) decrease in daily milk yield was not determined at any tested THI value (66-80). Taking into account that Simmentals produce significantly less milk than Holsteins, smaller drop in daily production due to the heat stress was expected, but the complete absence of production decline in older Simmentals needs to be further researched.

The decrease in daily milk yield due to the heat stress conditions in dairy cattle was intensively researched. For example, Bernabucci et al. (2010) and Collier et al. (2012) determined a decrease in milk production at $\mathrm{THI}=68$, while Bouraoui et al. (2002) in a Mediterranean climate observed it in conditions characterised by $\mathrm{THI} \geq 69$. Du Preez et al. (1990a, b) determined that dairy cows in Southern African conditions are affected by heat stress when THI values are higher than 72 . A significant decrease in the daily milk yield at $\mathrm{THI} \geq 72$ was also determined in Croatia (Gantner et al., 2011). Bohmanova et al. (2007) determined in the USA a different threshold values depending on the region (72 in Georgia, and 74 in Arizona). Same authors explained that the difference between the determined threshold values could be due to better adapted cows, farm management or special housing characteristics. Lambertz et al. (2014) determined highly significant drop in FCM (fat corrected milk) in Holsteins breed in four different housing systems with the highest drop occurring in traditional indoor housing systems.
Fluctuation of daily fat and protein content in milk due to heat stress concerning the parity class $\left(1^{\text {st }}, 2^{\text {nd }}, 3+\right)$ and breed (Holstein, Simmental) is presented in Table 3. Highly significant drop of daily fat and protein contents due to heat stress conditions was determined at all tested THI values (66-80) in both, the analysed breed and in all parity classes. In the first parity Holsteins, daily fat content was found to be in 0.191-0.214\%/day interval, in the second lactation Holsteins it was in 0.198-0.220\%/day interval, while the Holsteins in $3+$ parity experienced the highest decrease (range: 0.211-0.234\%/day).

The Simmental cows had higher drop in all parity classes, as follows: 0.232-0.269 \%/day, 0.238-0.273\%/day, 0.212-0.233\%/day intervals for first, second and third+ parity, respectively.

Similarly, Bouraoui et al. (2002) described a decrease of daily fat content (3.24 vs. $3.58 \%$ ), as well as a decrease of daily fat yield ( 0.68 vs. $0.48 \mathrm{~kg}$ ) during heat stress compared to normal condition that is in summer in regard to spring period.

The reduction in milk fat percentage associated with heat stress environments was also determined by Rodrigez et al. (1985) and Kadzere et al. (2002) who reported a decrease in the order of $39.7 \%$. In addition, Lambertz et al. (2014) found a significant decrease in a daily fat content with increasing THI values. Conversely, Knapp and Grummer (1991) did not find significant differences in milk fat content in cows under heat stress.

Regarding the daily protein content, similar trends as in daily fat content was determined. In the first parity Holsteins, decrease in daily protein content was found to be in 0.121-0.149\%/day interval, in the second lactation Holsteins it was in $0.136-0.166 \%$ /day interval, while the Holsteins in $3+$ parity experienced the highest decrease (range: 0.134-0.158 \%/day).

The Simmental cows had lower drop in all parity classes as follows: 0.110-0.140 \%/day, 0.116-0.140\%/day, 0.114-0.139\%/day intervals for first, second and third+ parity, respectively.

Similarly to these results, Bouraoui et al. (2002) found a decrease in a daily protein content ( 2.88 vs. $2.96 \%$ ), as well as decrease in the daily protein yield ( 0.56 vs. $0.43 \mathrm{~kg}$ ) during heat stress compared to normal condition found in summer in 
Table 3. Least square means of daily fat content regarding the given threshold values in accordance to the parity class and breed

\begin{tabular}{|c|c|c|c|c|c|c|}
\hline \multirow[b]{2}{*}{ ThHo } & \multicolumn{3}{|c|}{ Holstein } & \multicolumn{3}{|c|}{ Simmental } \\
\hline & Ls0 & Lsl & Difference & Ls0 & Lsl & Difference \\
\hline & \multicolumn{6}{|c|}{$1^{\text {st }}$ parity } \\
\hline THI66 & $4.13 \pm 0.006$ & $3.91 \pm 0.007$ & $0.211 \pm 0.003^{* * *}$ & $4.23 \pm 0.008$ & $3.99 \pm 0.009$ & $0.232 \pm 0.003^{* * *}$ \\
\hline THI67 & $4.12 \pm 0.006$ & $3.91 \pm 0.007$ & $0.214 \pm 0.003^{* * *}$ & $4.22 \pm 0.008$ & $3.99 \pm 0.009$ & $0.236 \pm 0.003^{* * *}$ \\
\hline THI68 & $4.12 \pm 0.006$ & $3.91 \pm 0.007$ & $0.207 \pm 0.003^{* * *}$ & $4.22 \pm 0.008$ & $3.98 \pm 0.009$ & $0.235 \pm 0.003^{* * *}$ \\
\hline THI69 & $4.11 \pm 0.006$ & $3.91 \pm 0.007$ & $0.203 \pm 0.003^{* * *}$ & $4.21 \pm 0.008$ & $3.97 \pm 0.009$ & $0.239 \pm 0.004^{* * *}$ \\
\hline THI70 & $4.11 \pm 0.006$ & $3.90 \pm 0.007$ & $0.208 \pm 0.004^{* * *}$ & $4.21 \pm 0.008$ & $3.96 \pm 0.009$ & $0.243 \pm 0.004^{* * *}$ \\
\hline THI71 & $4.10 \pm 0.006$ & $3.90 \pm 0.007$ & $0.205 \pm 0.004^{* * *}$ & $4.20 \pm 0.008$ & $3.95 \pm 0.009$ & $0.245 \pm 0.004^{* * *}$ \\
\hline THI72 & $4.10 \pm 0.006$ & $3.90 \pm 0.007$ & $0.198 \pm 0.004^{* *}$ & $4.20 \pm 0.008$ & $3.95 \pm 0.009$ & $0.249 \pm 0.004^{* * *}$ \\
\hline THI73 & $4.09 \pm 0.006$ & $3.90 \pm 0.007$ & $0.198 \pm 0.004^{* * *}$ & $4.19 \pm 0.008$ & $3.94 \pm 0.009$ & $0.251 \pm 0.004^{* * *}$ \\
\hline THI74 & $4.09 \pm 0.006$ & $3.88 \pm 0.007$ & $0.210 \pm 0.005^{* * *}$ & $4.19 \pm 0.008$ & $3.94 \pm 0.009$ & $0.255 \pm 0.005^{* * *}$ \\
\hline THI75 & $4.09 \pm 0.006$ & $3.88 \pm 0.008$ & $0.211 \pm 0.005^{* * *}$ & $4.19 \pm 0.008$ & $3.93 \pm 0.010$ & $0.260 \pm 0.005^{* * *}$ \\
\hline THI76 & $4.09 \pm 0.006$ & $3.88 \pm 0.008$ & $0.206 \pm 0.005^{* * *}$ & $4.18 \pm 0.008$ & $3.92 \pm 0.010$ & $0.260 \pm 0.005^{* * *}$ \\
\hline THI77 & $4.08 \pm 0.006$ & $3.88 \pm 0.008$ & $0.202 \pm 0.006^{* * *}$ & $4.18 \pm 0.008$ & $3.92 \pm 0.010$ & $0.260 \pm 0.006^{* * *}$ \\
\hline THI78 & $4.08 \pm 0.006$ & $3.87 \pm 0.009$ & $0.211 \pm 0.007^{* * *}$ & $4.18 \pm 0.008$ & $3.91 \pm 0.011$ & $0.269 \pm 0.007^{* * *}$ \\
\hline THI79 & $4.08 \pm 0.006$ & $3.88 \pm 0.009$ & $0.192 \pm 0.007^{* * *}$ & $4.17 \pm 0.008$ & $3.91 \pm 0.011$ & $0.267 \pm 0.007^{* * *}$ \\
\hline \multirow[t]{2}{*}{ THI80 } & $4.07 \pm 0.006$ & $3.88 \pm 0.010$ & $0.191 \pm 0.008^{* * *}$ & $4.17 \pm 0.008$ & $3.90 \pm 0.012$ & $0.271 \pm 0.008^{* * *}$ \\
\hline & \multicolumn{6}{|c|}{$2^{\text {nd }}$ parity } \\
\hline THI66 & $4.17 \pm 0.018$ & $3.95 \pm 0.019$ & $0.220 \pm 0.004^{* * *}$ & $4.22 \pm 0.010$ & $3.98 \pm 0.011$ & $0.238 \pm 0.004^{* * *}$ \\
\hline THI67 & $4.17 \pm 0.018$ & $3.95 \pm 0.019$ & $0.219 \pm 0.004^{* * *}$ & $4.21 \pm 0.010$ & $3.97 \pm 0.011$ & $0.240 \pm 0.004^{* * *}$ \\
\hline THI68 & $4.16 \pm 0.018$ & $3.95 \pm 0.019$ & $0.220 \pm 0.004^{* * *}$ & $4.21 \pm 0.010$ & $3.97 \pm 0.011$ & $0.239 \pm 0.004^{* * *}$ \\
\hline THI69 & $4.16 \pm 0.018$ & $3.94 \pm 0.019$ & $0.216 \pm 0.004^{* * *}$ & $4.20 \pm 0.010$ & $3.96 \pm 0.011$ & $0.242 \pm 0.004^{* * *}$ \\
\hline THI70 & $4.16 \pm 0.018$ & $3.94 \pm 0.019$ & $0.219 \pm 0.004^{* * *}$ & $4.20 \pm 0.010$ & $3.95 \pm 0.011$ & $0.245 \pm 0.004^{* * *}$ \\
\hline THI71 & $4.15 \pm 0.018$ & $3.93 \pm 0.019$ & $0.218 \pm 0.005^{* *}$ & $4.19 \pm 0.010$ & $3.94 \pm 0.011$ & $0.246 \pm 0.004^{* * *}$ \\
\hline THI72 & $4.15 \pm 0.018$ & $3.93 \pm 0.019$ & $0.214 \pm 0.005^{* * *}$ & $4.19 \pm 0.010$ & $3.94 \pm 0.011$ & $0.244 \pm 0.005^{* * *}$ \\
\hline THI73 & $4.14 \pm 0.018$ & $3.93 \pm 0.019$ & $0.209 \pm 0.005^{* *}$ & $4.18 \pm 0.010$ & $3.94 \pm 0.011$ & $0.244 \pm 0.005^{* * *}$ \\
\hline THI74 & $4.14 \pm 0.019$ & $3.93 \pm 0.019$ & $0.210 \pm 0.006^{* *}$ & $4.18 \pm 0.010$ & $3.93 \pm 0.011$ & $0.246 \pm 0.005^{* * *}$ \\
\hline THI75 & $4.14 \pm 0.019$ & $3.92 \pm 0.019$ & $0.213 \pm 0.006^{* *}$ & $4.18 \pm 0.010$ & $3.92 \pm 0.012$ & $0.252 \pm 0.006^{* * *}$ \\
\hline THI76 & $4.13 \pm 0.019$ & $3.92 \pm 0.019$ & $0.208 \pm 0.006^{* *}$ & $4.17 \pm 0.010$ & $3.92 \pm 0.012$ & $0.255 \pm 0.006^{* * *}$ \\
\hline THI77 & $4.13 \pm 0.019$ & $3.92 \pm 0.020$ & $0.211 \pm 0.007^{* * *}$ & $4.17 \pm 0.010$ & $3.91 \pm 0.012$ & $0.264 \pm 0.007^{* * *}$ \\
\hline THI78 & $4.13 \pm 0.019$ & $3.92 \pm 0.020$ & $0.211 \pm 0.008^{* * *}$ & $4.17 \pm 0.010$ & $3.90 \pm 0.013$ & $0.270 \pm 0.007^{* * *}$ \\
\hline THI79 & $4.12 \pm 0.019$ & $3.93 \pm 0.020$ & $0.199 \pm 0.009^{* * *}$ & $4.17 \pm 0.010$ & $3.89 \pm 0.013$ & $0.273 \pm 0.008^{* * *}$ \\
\hline \multirow[t]{2}{*}{ THI80 } & $4.12 \pm 0.019$ & $3.93 \pm 0.021$ & $0.198 \pm 0.010^{* * *}$ & $4.16 \pm 0.010$ & $3.90 \pm 0.014$ & $0.266 \pm 0.009^{* * *}$ \\
\hline & \multicolumn{6}{|c|}{$3+$ parity } \\
\hline THI66 & $4.14 \pm 0.007$ & $3.91 \pm 0.008$ & $0.234 \pm 0.004^{* * *}$ & $4.18 \pm 0.013$ & $3.97 \pm 0.013$ & $0.212 \pm 0.002^{* * *}$ \\
\hline THI67 & $4.14 \pm 0.007$ & $3.91 \pm 0.008$ & $0.229 \pm 0.004^{* *}$ & $4.17 \pm 0.013$ & $3.96 \pm 0.013$ & $0.213 \pm 0.003^{* * *}$ \\
\hline THI68 & $4.13 \pm 0.007$ & $3.90 \pm 0.008$ & $0.226 \pm 0.004^{* *}$ & $4.17 \pm 0.013$ & $3.96 \pm 0.013$ & $0.212 \pm 0.003^{* * *}$ \\
\hline THI69 & $4.12 \pm 0.007$ & $3.90 \pm 0.008$ & $0.220 \pm 0.004^{* * *}$ & $4.16 \pm 0.013$ & $3.95 \pm 0.013$ & $0.213 \pm 0.003^{* * *}$ \\
\hline THI70 & $4.12 \pm 0.007$ & $3.89 \pm 0.008$ & $0.226 \pm 0.004^{* * *}$ & $4.16 \pm 0.013$ & $3.94 \pm 0.013$ & $0.215 \pm 0.003^{* * *}$ \\
\hline THI71 & $4.11 \pm 0.007$ & $3.89 \pm 0.008$ & $0.227 \pm 0.004^{* * *}$ & $4.15 \pm 0.013$ & $3.94 \pm 0.013$ & $0.216 \pm 0.003^{* * *}$ \\
\hline THI72 & $4.11 \pm 0.007$ & $3.89 \pm 0.008$ & $0.221 \pm 0.005^{* * *}$ & $4.15 \pm 0.013$ & $3.93 \pm 0.013$ & $0.215 \pm 0.003^{* * *}$ \\
\hline THI73 & $4.11 \pm 0.007$ & $3.89 \pm 0.008$ & $0.220 \pm 0.005^{* *}$ & $4.15 \pm 0.013$ & $3.93 \pm 0.013$ & $0.215 \pm 0.003^{* * *}$ \\
\hline THI74 & $4.10 \pm 0.007$ & $3.88 \pm 0.009$ & $0.220 \pm 0.005^{* * *}$ & $4.14 \pm 0.013$ & $3.93 \pm 0.013$ & $0.216 \pm 0.004^{* * *}$ \\
\hline THI75 & $4.10 \pm 0.007$ & $3.88 \pm 0.009$ & $0.218 \pm 0.006^{* *}$ & $4.14 \pm 0.013$ & $3.92 \pm 0.013$ & $0.218 \pm 0.004^{* * *}$ \\
\hline THI76 & $4.10 \pm 0.007$ & $3.88 \pm 0.009$ & $0.215 \pm 0.006^{* *}$ & $4.14 \pm 0.013$ & $3.92 \pm 0.013$ & $0.222 \pm 0.004^{* * *}$ \\
\hline THI77 & $4.09 \pm 0.007$ & $3.87 \pm 0.009$ & $0.222 \pm 0.007^{* *}$ & $4.14 \pm 0.013$ & $3.91 \pm 0.013$ & $0.224 \pm 0.005^{* * *}$ \\
\hline THI78 & $4.09 \pm 0.007$ & $3.87 \pm 0.010$ & $0.217 \pm 0.007^{* * *}$ & $4.13 \pm 0.013$ & $3.90 \pm 0.014$ & $0.233 \pm 0.005^{* * *}$ \\
\hline THI79 & $4.09 \pm 0.007$ & $3.87 \pm 0.011$ & $0.213 \pm 0.008^{* * *}$ & $4.13 \pm 0.013$ & $3.91 \pm 0.014$ & $0.226 \pm 0.006^{* * *}$ \\
\hline THI80 & $4.09 \pm 0.007$ & $3.87 \pm 0.012$ & $0.211 \pm 0.009^{* * *}$ & $4.13 \pm 0.013$ & $3.90 \pm 0.014$ & $0.233 \pm 0.006^{* * *}$ \\
\hline
\end{tabular}

* ThHo - given threshold value; 0 - class under, and 1 - class above the given threshold value 
Table 4. Least square means of daily protein content regarding the given threshold value in accordance to the parity class and breed

\begin{tabular}{|c|c|c|c|c|c|c|}
\hline \multirow[b]{2}{*}{ ThHo } & \multicolumn{3}{|c|}{ Holstein } & \multicolumn{3}{|c|}{ Simmental } \\
\hline & Ls0 & Lsl & Difference & Ls0 & Lsl & Difference \\
\hline & \multicolumn{6}{|c|}{$1^{\text {st }}$ parity } \\
\hline THI66 & $3.43 \pm 0.003$ & $3.31 \pm 0.003$ & $0.121 \pm 0.001^{* * *}$ & $3.47 \pm 0.004$ & $3.36 \pm 0.004$ & $0.110 \pm 0.001^{* * *}$ \\
\hline THI67 & $3.43 \pm 0.003$ & $3.30 \pm 0.003$ & $0.123 \pm 0.001^{* * *}$ & $3.47 \pm 0.004$ & $3.36 \pm 0.004$ & $0.114 \pm 0.002^{* * *}$ \\
\hline THI68 & $3.43 \pm 0.003$ & $3.30 \pm 0.003$ & $0.123 \pm 0.001^{* * *}$ & $3.47 \pm 0.004$ & $3.35 \pm 0.004$ & $0.115 \pm 0.002^{* * *}$ \\
\hline THI69 & $3.42 \pm 0.003$ & $3.30 \pm 0.003$ & $0.124 \pm 0.001^{* * *}$ & $3.47 \pm 0.004$ & $3.35 \pm 0.004$ & $0.116 \pm 0.002^{* * *}$ \\
\hline THI70 & $3.42 \pm 0.003$ & $3.30 \pm 0.003$ & $0.126 \pm 0.001^{* * *}$ & $3.46 \pm 0.004$ & $3.35 \pm 0.004$ & $0.118 \pm 0.002^{* * *}$ \\
\hline THI71 & $3.42 \pm 0.003$ & $3.29 \pm 0.003$ & $0.128 \pm 0.002^{* * *}$ & $3.46 \pm 0.004$ & $3.34 \pm 0.004$ & $0.120 \pm 0.002^{* * *}$ \\
\hline THI72 & $3.42 \pm 0.003$ & $3.28 \pm 0.003$ & $0.132 \pm 0.002^{* * *}$ & $3.46 \pm 0.004$ & $3.34 \pm 0.004$ & $0.121 \pm 0.002^{* * *}$ \\
\hline THI73 & $3.42 \pm 0.003$ & $3.28 \pm 0.003$ & $0.134 \pm 0.002^{* * *}$ & $3.46 \pm 0.004$ & $3.33 \pm 0.004$ & $0.124 \pm 0.002^{* * *}$ \\
\hline THI74 & $3.41 \pm 0.003$ & $3.28 \pm 0.003$ & $0.136 \pm 0.002^{* * *}$ & $3.46 \pm 0.004$ & $3.33 \pm 0.004$ & $0.125 \pm 0.002^{* * *}$ \\
\hline THI75 & $3.41 \pm 0.003$ & $3.27 \pm 0.003$ & $0.137 \pm 0.002^{* * *}$ & $3.46 \pm 0.004$ & $3.33 \pm 0.004$ & $0.126 \pm 0.002^{* * *}$ \\
\hline THI76 & $3.41 \pm 0.003$ & $3.27 \pm 0.003$ & $0.137 \pm 0.002^{* * *}$ & $3.45 \pm 0.004$ & $3.33 \pm 0.004$ & $0.129 \pm 0.002^{* * *}$ \\
\hline THI77 & $3.41 \pm 0.003$ & $3.27 \pm 0.003$ & $0.140 \pm 0.002^{* * *}$ & $3.45 \pm 0.004$ & $3.32 \pm 0.005$ & $0.132 \pm 0.003^{* * *}$ \\
\hline THI78 & $3.41 \pm 0.003$ & $3.27 \pm 0.004$ & $0.136 \pm 0.003^{* * *}$ & $3.45 \pm 0.004$ & $3.32 \pm 0.005$ & $0.135 \pm 0.003^{* * *}$ \\
\hline THI79 & $3.40 \pm 0.003$ & $3.26 \pm 0.004$ & $0.140 \pm 0.003^{* * *}$ & $3.45 \pm 0.004$ & $3.31 \pm 0.005$ & $0.137 \pm 0.003^{* * *}$ \\
\hline \multirow[t]{2}{*}{ THI80 } & $3.40 \pm 0.003$ & $3.25 \pm 0.004$ & $0.149 \pm 0.003^{* * *}$ & $3.45 \pm 0.004$ & $3.31 \pm 0.005$ & $0.140 \pm 0.004^{* * *}$ \\
\hline & \multicolumn{6}{|c|}{$2^{\text {nd }}$ parity } \\
\hline THI66 & $3.44 \pm 0.008$ & $3.31 \pm 0.008$ & $0.136 \pm 0.002^{* * *}$ & $3.51 \pm 0.005$ & $3.39 \pm 0.005$ & $0.116 \pm 0.002^{* * *}$ \\
\hline THI67 & $3.44 \pm 0.008$ & $3.30 \pm 0.008$ & $0.139 \pm 0.002^{* * *}$ & $3.51 \pm 0.005$ & $3.39 \pm 0.005$ & $0.119 \pm 0.002^{* * *}$ \\
\hline THI68 & $3.44 \pm 0.008$ & $3.30 \pm 0.008$ & $0.139 \pm 0.002^{* * *}$ & $3.51 \pm 0.005$ & $3.38 \pm 0.005$ & $0.120 \pm 0.002^{* * *}$ \\
\hline THI69 & $3.43 \pm 0.008$ & $3.29 \pm 0.008$ & $0.140 \pm 0.002^{* * *}$ & $3.50 \pm 0.005$ & $3.38 \pm 0.005$ & $0.121 \pm 0.002^{* * *}$ \\
\hline THI70 & $3.43 \pm 0.008$ & $3.29 \pm 0.008$ & $0.143 \pm 0.002^{* * *}$ & $3.50 \pm 0.005$ & $3.38 \pm 0.005$ & $0.121 \pm 0.002^{* * *}$ \\
\hline THI71 & $3.43 \pm 0.008$ & $3.28 \pm 0.008$ & $0.146 \pm 0.002^{* * *}$ & $3.50 \pm 0.005$ & $3.37 \pm 0.005$ & $0.123 \pm 0.002^{* * *}$ \\
\hline THI72 & $3.43 \pm 0.008$ & $3.28 \pm 0.008$ & $0.149 \pm 0.002^{* * *}$ & $3.49 \pm 0.005$ & $3.37 \pm 0.005$ & $0.123 \pm 0.002^{* * *}$ \\
\hline THI73 & $3.42 \pm 0.008$ & $3.27 \pm 0.008$ & $0.150 \pm 0.002^{* * *}$ & $3.49 \pm 0.005$ & $3.37 \pm 0.005$ & $0.124 \pm 0.002^{* * *}$ \\
\hline THI74 & $3.42 \pm 0.008$ & $3.27 \pm 0.008$ & $0.150 \pm 0.002^{* * *}$ & $3.49 \pm 0.005$ & $3.37 \pm 0.005$ & $0.125 \pm 0.002^{* * *}$ \\
\hline THI75 & $3.42 \pm 0.008$ & $3.27 \pm 0.008$ & $0.153 \pm 0.002^{* * *}$ & $3.49 \pm 0.005$ & $3.36 \pm 0.005$ & $0.125 \pm 0.003^{* * *}$ \\
\hline THI76 & $3.42 \pm 0.008$ & $3.27 \pm 0.008$ & $0.150 \pm 0.003^{* * *}$ & $3.49 \pm 0.005$ & $3.36 \pm 0.005$ & $0.128 \pm 0.003^{* * *}$ \\
\hline THI77 & $3.42 \pm 0.008$ & $3.26 \pm 0.008$ & $0.154 \pm 0.003^{* * *}$ & $3.49 \pm 0.005$ & $3.35 \pm 0.006$ & $0.135 \pm 0.003^{* * *}$ \\
\hline THI78 & $3.41 \pm 0.008$ & $3.26 \pm 0.008$ & $0.156 \pm 0.003^{* * *}$ & $3.49 \pm 0.005$ & $3.35 \pm 0.006$ & $0.139 \pm 0.003^{* * *}$ \\
\hline THI79 & $3.41 \pm 0.008$ & $3.25 \pm 0.009$ & $0.158 \pm 0.004^{* * *}$ & $3.48 \pm 0.005$ & $3.34 \pm 0.006$ & $0.140 \pm 0.004^{* * *}$ \\
\hline \multirow[t]{2}{*}{ THI80 } & $3.41 \pm 0.008$ & $3.25 \pm 0.009$ & $0.166 \pm 0.004^{* * *}$ & $3.48 \pm 0.005$ & $3.34 \pm 0.006$ & $0.140 \pm 0.004^{* * *}$ \\
\hline & \multicolumn{6}{|c|}{$3+$ parity } \\
\hline THI66 & $3.39 \pm 0.003$ & $3.25 \pm 0.003$ & $0.134 \pm 0.001^{* * *}$ & $3.45 \pm 0.005$ & $3.34 \pm 0.006$ & $0.114 \pm 0.001^{* * *}$ \\
\hline THI67 & $3.39 \pm 0.003$ & $3.25 \pm 0.003$ & $0.135 \pm 0.002^{* * *}$ & $3.45 \pm 0.005$ & $3.33 \pm 0.006$ & $0.115 \pm 0.001^{* * *}$ \\
\hline THI68 & $3.38 \pm 0.003$ & $3.25 \pm 0.003$ & $0.135 \pm 0.002^{* * *}$ & $3.45 \pm 0.005$ & $3.33 \pm 0.006$ & $0.117 \pm 0.001^{* * *}$ \\
\hline THI69 & $3.38 \pm 0.003$ & $3.24 \pm 0.003$ & $0.135 \pm 0.002^{* * *}$ & $3.44 \pm 0.006$ & $3.33 \pm 0.006$ & $0.118 \pm 0.001^{* * *}$ \\
\hline THI70 & $3.38 \pm 0.003$ & $3.24 \pm 0.003$ & $0.138 \pm 0.002^{* * *}$ & $3.44 \pm 0.006$ & $3.32 \pm 0.006$ & $0.119 \pm 0.001^{* * *}$ \\
\hline THI71 & $3.37 \pm 0.003$ & $3.23 \pm 0.003$ & $0.140 \pm 0.002^{* * *}$ & $3.44 \pm 0.006$ & $3.32 \pm 0.006$ & $0.120 \pm 0.001^{* * *}$ \\
\hline THI72 & $3.37 \pm 0.003$ & $3.23 \pm 0.003$ & $0.143 \pm 0.002^{* * *}$ & $3.44 \pm 0.006$ & $3.32 \pm 0.006$ & $0.121 \pm 0.001^{* * *}$ \\
\hline THI73 & $3.37 \pm 0.003$ & $3.23 \pm 0.003$ & $0.143 \pm 0.002^{* * *}$ & $3.44 \pm 0.006$ & $3.31 \pm 0.006$ & $0.123 \pm 0.001^{* * *}$ \\
\hline THI74 & $3.37 \pm 0.003$ & $3.22 \pm 0.004$ & $0.144 \pm 0.002^{* * *}$ & $3.43 \pm 0.006$ & $3.31 \pm 0.006$ & $0.124 \pm 0.002^{* * *}$ \\
\hline THI75 & $3.37 \pm 0.003$ & $3.22 \pm 0.004$ & $0.146 \pm 0.002^{* * *}$ & $3.43 \pm 0.006$ & $3.31 \pm 0.006$ & $0.123 \pm 0.002^{* * *}$ \\
\hline THI76 & $3.36 \pm 0.003$ & $3.22 \pm 0.004$ & $0.146 \pm 0.002^{* * *}$ & $3.43 \pm 0.006$ & $3.31 \pm 0.006$ & $0.125 \pm 0.002^{* * *}$ \\
\hline THI77 & $3.36 \pm 0.003$ & $3.21 \pm 0.004$ & $0.148 \pm 0.003^{* * *}$ & $3.43 \pm 0.006$ & $3.30 \pm 0.006$ & $0.131 \pm 0.002^{* * *}$ \\
\hline THI78 & $3.36 \pm 0.003$ & $3.21 \pm 0.004$ & $0.149 \pm 0.003^{* * *}$ & $3.43 \pm 0.006$ & $3.29 \pm 0.006$ & $0.135 \pm 0.002^{* * *}$ \\
\hline THI79 & $3.36 \pm 0.003$ & $3.21 \pm 0.004$ & $0.149 \pm 0.003^{* * *}$ & $3.43 \pm 0.006$ & $3.29 \pm 0.006$ & $0.135 \pm 0.002^{* * *}$ \\
\hline THI80 & $3.36 \pm 0.003$ & $3.20 \pm 0.005$ & $0.158 \pm 0.004^{* * *}$ & $3.43 \pm 0.006$ & $3.29 \pm 0.006$ & $0.139 \pm 0.003^{* * *}$ \\
\hline
\end{tabular}

* ThHo - given threshold value; 0 - class under, and 1 - class above the given threshold value 
regard to spring period. The reduction in milk protein percentages associated with heat stress environments was also determined by Rodrigez et al. (1985) and Kadzere et al. (2002) who reported a decrease for $16.9 \%$. Knapp and Grummer (1991) explained the decrease of protein with increasing maximum daily temperature by a decreased dry matter and energy intake. In addition, Lambertz et al. (2014) found a significant decrease in the daily protein content with increasing THI values in Holsteins housed indoors.

\section{Conclusion}

Based on the analyses conducted within the present study, it could be concluded that the threshold values for daily milk yield were highly dependant on the parity and breed (Holsteins: 68, 69 and 72 for $1^{\text {st }}, 2^{\text {nd }}, 3+$ parity; Simmentals: 77 for $\left.1^{\text {st }}\right)$. Also, the daily fat and protein contents showed a significant decrease due to heat stress conditions (THI in 6680) in both, Holstein and Simmental cows, and in all parity classes as well. Multiparous cows seemed to be more resistant to heat stress than primiparous, while Simmental cows seemed to be more resistant to heat stress than Holsteins. However, the question still remains are Simmentals more heat stress resistant? Is the supposed resistance found in this research mainly a consequence of the lower production level of Simmentals or are they genetically more appropriate for the expected challenges in the future milk production? These questions will be answered by subsequent researches.

\section{Razlike u rezistentnosti na toplinski stres ovisno o pasmini mliječnih goveda}

\section{Sažetak}

U svjetlu sve izraženijih te bržih klimatskih promjena širom svijeta, te $u$ cilju redukcije financijskih gubitaka mliječnih farmi i osiguravanja održivog poslovanja, nužnost implementacije uzgojnih vrijednosti za rezistentnost na toplinski stres u uzgojne strategije, biva sve izraženija. Procjena uzgojnih vrijednosti podrazumijeva prethodnu determinaciju threshold vrijednosti. Stoga je cilj ovoga rada bio utvrditi threshold vrijednost za dnevnu količinu mlijeka, dnevni sadržaj mliječne masti te bjelančevina za holstein i simentalsku pasminu na mliječnim farmama u Hrvatskoj. S tim je ciljem analizirano 1.070.554 testday zapisa 70.135 holstein krava uzgajanih na 5679 farmi te 1.300 .683 test-day zapisa 86.013 krava simentalske pasmine uzgajanih na 8827 farmi u Hrvatskoj zajedno s podacima o ambijentalnoj temperaturi te relativnoj vlazi na farmi prikupljenih tijekom redovne kontrole mliječnosti u razdoblju od siječnja 2005. do prosinca 2012. Temeljem provedene analize mogu se donijeti slijedeći zaključci: threshold vrijednost za dnevnu količinu mlijeka uvelike ovisi o redoslijedu laktacije te pasmini (holstein: 68, 69 te 72 za 1., 2., te 3+ laktaciju; simentalska: 77 za 1); dnevni sadržaj mliječne masti te bjelančevina statistički visoko signifikantno opada uslijed toplinskog stresa (THI jednak 66-80) u obje pasmine te u svim laktacijama; višetelke su rezistentnije na toplinski stres $\mathrm{u}$ odnosu na prvotelke; krave simentalske pasmine su rezistentnije na toplinski stres $\mathrm{u}$ odnosu na holstein.

\footnotetext{
Ključne riječi: mliječna goveda, holstein, simentalska, toplinski stres, threshold vrijednost
} 


\section{References}

1. Aguilar, I., Misztal, I., Tsuruta, S. (2009): Genetic components of heat stress for dairy cattle with multiple lactation. Journal of Dairy Science 92, 5702-5711. https://doi.org/10.3168/jds.2008-1928

2. Aharoni, Y., Brosh, A., Ezra, E. (1999): Effect of heat load and photoperiod on milk yield and composition in three dairy herds in Israel. Animal Science 69, 37-47. https://doi.org/10.1017/S1357729800051079

3. Berman, A. (2005): Estimates of heat stress relief needs for Holstein dairy cows. Journal of Animal Science 83, 1377-1384. https://doi.org/10.2527/2005.8361377x

4. Bernabucci, U., Lacetera, N., Baumgard, L.H., Rhoads, R.P., Ronchi, B., Nardone, A. (2010): Metabolic and hormonal acclimation to heat stress in domestic ruminants. Animal 4, 1167-1183. https://doi.org/10.1017/S175173111000090X

5. Bohmanova, J. (2006): Studies on genetics of heat stress in US Holsteins. PhD thesis, University of Georgia, Athens, GA, USA.

6. Bohmanova, J., Misztal, I., Cole, J.B. (2007): Temperature-humidity indices as indicators of milk production losses due to heat stress. Journal of Dairy Science 90, 1947-1956. https://doi.org/10.3168/jds.2006-513

7. Bouraoui, R., Lahmar, M., Majdoub, A., Djemali, M., Belyea, R. (2002): The relationship of temperature humidity-index with milk production of dairy cows in a Mediterranean climate. Animal Research 51, 479-491. https://doi.org/10.1051/animres:2002036

8. Collier, R.J., Dahl, G.E., VanBaale, M.J. (2006): Major advances associated with environmental effects on dairy cattle. Journal of Dairy Science 89, 1244-1253. https://doi.org/10.3168/jds.S0022-0302(06)72193-2

9. Collier, Robert J., Hall, Laun, W. (2012): Quantifying heat stress and its impact on metabolism and performance. Department of Animal Sciences. University of Arizona.

10. Correa-Calderon, A., Armstrong, D., Ray, D., DeNise, S., Enns, M., Howison, C. (2004): Thermoregulatory responses of Holstein and Brown Swiss heatstressed dairy cows to two different cooling systems. International Journal of Biometeorology 48, 142-148. https://doi.org/10.1007/s00484-003-0194-y

11. Dunn, R.J.H., Mead, N.E., Willett, K.M., Parker, D.E. (2014): Analysis of heat stress in UK dairy cattle and impact on milk yields Environ. Research Letters 9, 064006 (11 pp).

12. Du Preez, J.H., Giesecke, W.H., Hattingh, P.J. (1990a): Heat stress in dairy cattle and other livestock under Southern African conditions. I. Temperature-humidity index mean values during the four main seasons. Onderstepoort Journal of Veterinary Research 57, 77-86.
13. Du Preez, J.H., Hatting, P.J., Giesecke, W.H., Eisenberg, B.E. (1990b): Heat stress in dairy cattle and other livestock under Southern African conditions. III. Monthly temperature-humidity index mean values and their significance in the performance of dairy cattle. Onderstepoort. Journal of Veterinary Research 57, 243-248.

14. Freitas, M., Misztal, I., Bohmanova, J., Torres, R. (2006): Regional differences in heat stress in US Holsteins. Proc. $8^{\text {th }}$ World Congr. Genet. Appl. Livest. Prod. Commun. 01-11. Istituto Prociencia, Belo Horizonte, Brazil.

15. Gantner, V., Mijić, P., Kuterovac, K., Solić, D., Gantner, R. (2011): Temperature-humidity index values and their significance on the daily production of dairy cattle. Mljekarstvo 61 (1), 56-63.

16. Gauly, M., Bollwein, H., Breves, G., Brügemann, K., Dänicke, K., Das, G., Demeler, G., Hansen, H., Isselstein, J., König, S., Lohölter, M., Martinsohn, M., Meyer, U., Potthoff, M., Sanker, C., Schröder, B., Wrage, N., Meibaum, B., von Samson-Himmelstjerna, G., Stinshoff, H., Wrenzycki, C. (2013): Future consequences and challenges for dairy cow production systems arising from climate change in Central Europe - a review. Animal 7, 843-859. https://doi.org/10.1017/S1751731112002352

17. Gira - Consultancy \& Research Prospective et Strategie (2012): World and EU dairy through 2016. http:// ec.europa.eu/agriculture/sites/agriculture/files/milk / background/jm-2012-12-12/01-gira_en.pdf

18. Intergovernmental Panel on Climate Change - IPCC (2007): Climate Change 2007: The Physical Science Basis. Contribution of Working Group I to the Fourth Assessment Report of the Intergovernmental Panel on Climate Change. Cambridge University Press, Cambridge/ New York, NY, USA.

19. ICAR - International Committee for Animal Recording (2003): Guidelines approved by the General Assembly held in Interlaken, Switzerland, on 30 May 2002, Roma, $19-39$.

20. Kadzere, C.T., Murphy, M.R., Silanikove, N., Maltz, E. (2002): Heat Stress In Lactating Dairy Cows: A Review. Livestock of Production Science 77, 59-91. https://doi.org/10.1016/S0301-6226(01)00330-X

21. Kibler, H.H. (1964): Environmental physiology and shelter engineering. LXVII. Thermal effects of various temperature-humidity combinations on Holstein cattle as measured by eight physiological responses. Research Bulletin Missouri Agriculture Expanded Station, 862.

22. Knapp, D.M., Grummer, R.R. (1991): Response of lactating dairy cows to fat supplementation during heat stress. Journal of Dairy Science 74, 2573-2579. https://doi.org/10.3168/jds.S0022-0302(91)78435-X

23. Lambertz, C., Sanker, C., Gauly, M. (2014): Climatic effects on milk production traits and somatic cell score in lactating Holstein-Friesian cows in different housing systems. American Dairy Science Association. Journal of Dairy Science 97, (1), 319-329. https://doi.org/10.3168/jds.2013-7217 
24. Nardone, A., Ronchi, B., Lacetera, N., Ranieri, M.S., Bernabucci, U. (2010): Effects of climate changes on animal production and sustainability of livestock systems. Livestock Science 130, 57-69. https://doi.org/10.1016/j.livsci.2010.02.011

25. Ravagnolo, O., Misztal, I. (2002a): Studies on genetics of heat tolerance in dairy cattle with reduced weather information via cluster analysis. Journal of Dairy Science 85, 1586-1589. https://doi.org/10.3168/jds.S0022-0302(02)74228-8

26. Ravagnolo, O., Misztal, I. (2002b): Effect of heat stress on nonreturn rate in Holstein cows: Genetic analysis. Journal of Dairy Science 85, 3092-3100. https://doi.org/10.3168/jds.S0022-0302(02)74396-8

27. Ravagnolo, O., Misztal, I., Hoogenboom, G. (2000): Genetic component of heat stress in dairy cattle, development of heat indeks function. Journal of Dairy Science 83, 2120-2125. https://doi.org/10.3168/jds.S0022-0302(00)75094-6

28. Reiczigel, J., Solymosi, N., Könyves, L., Maróti-Agóts, A., Kern, A., Bartyik, J. (2009): Examination of heat stress caused milk production loss by the use of temperature-humidity indices. Magy Allatorv 131, 137-144.

29. Rodriguez, L.W., Mekonnen, G., Wilcox, C.J., Martin, F.G., Krienk, W.A. (1985): Effects of relative humidity, maximum and minimum temperature, pregnancy and stage of lactation on milkcomposition and yield. Journal of Dairy Science 68, 973-978. https://doi.org/10.3168/jds.S0022-0302(85)80917-6
30. Romaniuk, W., Overby, T. (2005): A guide to cattle housing systems (in Polish). IBMER. Warsaw.

31. SAS User's Guide (2000): Version 8.2 Edition. SAS Institute Inc. Cary, NC.

32. St-Pierre, N.R., Cobanov, B., Schnitkey, G. (2003): Economic loses from heat stress by US livestock industries. Journal of Dairy Science 86, 52-77. https://doi.org/10.3168/jds.S0022-0302(03)74040-5

33. Vitali, A., Sagnalini, M., Bertocchi, L., Bernabucci, U., Nardone, A., Lacetera, N. (2009): Seasonal pattern of mortality and relationships between mortality and temperature humidity index in dairy cows. Journal of Dairy Science 92, 3781-3790. https://doi.org/10.3168/jds.2009-2127

34. Yousef, M.K. (1985): Stress Physiology in Livestock. Vol. 1. CRC Press, Boca Raton. Str. 67-73.

35. West, J.W. (2003): Effects of heat-stress on production in dairy cattle. Journal of Dairy Science 86, 2131-2144.

36. West, J.W., Hill, G.M., Fernandez, J.M., Mandebvu, P., Mullinix, B.G. (1999): Effect of dietary fiber on intake, milk yield, and digestion by lactating dairy cows during cool or hot, humid weather. Journal of Dairy Science 82, 2455-2465. https://doi.org/10.3168/jds.S0022-0302(99)75497-4 